\title{
LAS HERRAMIENTAS INFORMÁTICAS DE EDICIÓN EN LA FORMACIÓN DE LOS FUTUROS PROFESIONALES DE LA COMUNICACIÓN SOCIAL EN LA FACSO DE LA UNIVERSIDAD DE GUAYAQUIL.
}

\begin{abstract}
EDITING TOOLS IN THE FORMATION OF THE PROFESSIONAL FUTURES OF SOCIAL COMMUNICATION IN THE FACSO OF UNIVERSITY OF GUAYAQUIL.
\end{abstract}

Janeth Pilar Díaz Vera, Mgs. Magíster en Educación Informática (Ecuador). Docente de la Facultad de Filosofía de la Universidad de Guayaquil (Ecuador). orcid.org/0000-0001-8750-0216 janeth.diazv@ug.edu.ec

Delia Silvia Peña Hojas, Ph. D. Doctora en Ciencias de la Educación (Cuba). Docente de la Facultad de Medicina de la Universidad de Guayaquil (Ecuador). dphojas@hotmail.com

Dr. Guido Niman Moreno Córdova, Mgs. Magíster en Gerencia de Salud y Desarrollo local (Ecuador). Docente de la Facultad de Medicina de la Universidad Católica Santiago de Guayaquil (Ecuador). guimocordr@hotmail.com

\section{Lcdo. David Augusto Díaz Vera}

Licenciado en Ciencias de la Educación mención Educación Primaria (Ecuador). Docente de la Facultad de Filosofía de la Universidad de Guayaquil y del Ministerio de Educación en la Institución de Educación Básica "Said Hanna” (Ecuador). david.diaz@educacion.gob.ec

\section{ARTíCULO DE INVESTIGACIÓN}

Recibido: 14 de enero de 2019.

Aceptado: 23 de abril de 2019. 


\section{RESUMEN}

La presente investigación se enmarca dentro del tema: las herramientas informáticas de edición en la formación de los futuros profesionales de la comunicación social (sección nocturna) de la Facultad de Comunicación Social (FACSO). En la estructura se hace un recorrido histórico, epistemológico y sociológico donde se identifica la necesidad de poseer el conocimiento y desarrollo de habilidades y destrezas sobre los programas de edición para el óptimo ejercicio profesional en el entorno comunicacional. La metodología utilizada es un diseño cuantitativo con un alcance explicativo y descriptivo donde se aplican los métodos: histórico y lógico, análisis y síntesis, inductivo y deductivo con la técnica de la encuesta. La población abarca los estudiantes del sexto semestre, sección nocturna, de la carrera de Comunicación Social quienes fueron los beneficiarios de este estudio; finalmente la muestra la conforma el cincuenta por ciento de la población por lo que se puede afirmar que es representativa. El instrumento de investigación utilizados tanto para la entrevista como para la encuesta fue un cuestionario de preguntas elaboradas previamente para su aplicación. La propuesta consistió en diseñar un plan estratégico de comunicación que favorezca el aprendizaje y dominio de las herramientas informáticas de edición mediante la realización de talleres prácticos para que los estudiantes y futuros comunicadores sociales adquieran las competencias necesarias para su formación profesional.

Palabras clave: Herramientas, informática, edición, comunicación, formación profesional.

\section{ABSTRACT}

This research is framed within the theme: the editing tools in the training of social communication of future professionals (night section) of the Faculty of Social Communication (FACSO). In the structure there is a historical, epistemological and sociological journey where the need to possess the knowledge and development of skills and abilities on the editing programs for the optimal professional practice in the communication environment is identified. The methodology used is a quantitative design with an explanatory and descriptive scope where the applied methods are: historical and logical, analysis and synthesis, inductive and deductive with the technique of the survey. The population includes the students of the sixth semester, night section, of the career of Social Communication who were the beneficiaries of this study; finally, the sample is made 
up fifty percent of the population, so it can be said that it is representative. The research instruments used for both the interview and the survey was a questionnaire of questions previously prepared for its application. The proposal consisted in the design of a strategic communication plan that favors the learning and mastery of the editing computer tools through the realization of practical workshops that students and future social communicators acquire the necessary skills for their professional training.

Keywords: Tools, computing, publishing, communication, professional training.

\section{INTRODUCCIÓN}

La labor periodística en la actualidad gira sobre un entorno digital, donde los futuros profesionales de la comunicación deben estar a la par con las nuevas tecnologías y requerimientos de los medios de comunicación para desarrollar con óptimos resultados sus tareas diarias. El presente trabajo se enmarca en el dominio de las herramientas informáticas de edición por parte de los profesionales de Comunicación Social de FACSO en el período 2016-2017.

Se planteó la siguiente interrogante como punto de partida, ¿Por qué las insuficiencias en el manejo de las herramientas informáticas de edición en la formación de los estudiantes de Comunicación Social en el período 2016-2017? Los objetivos planteados para el análisis y realización de la investigación se enfocan en caracterizar las deficiencias en la formación de los fututos profesionales de la Comunicación, relacionado con las herramientas informáticas de edición; sistematizar los contenidos sobre las herramientas de edición de audio, sonido y texto. Como objetivos específicos se tiene:

- Caracterizar las deficiencias en el uso de las herramientas informáticas de edición que poseen los estudiantes del sexto semestre de la carrera de Comunicación Social en el período 2015-2016.

- Sistematizar los conceptos sobre las herramientas informáticas de edición tanto de audio (Adobe Audition), como de video (Adobe Premier) y texto (Adobe InDesing). 
- Diseñar un Plan Comunicacional que favorezca el aprendizaje y manejo de las herramientas informáticas de edición en los estudiantes de la carrera de comunicación social en el período 2015-2016.

Durante el proceso de investigación se planteó la siguiente hipótesis: con la aplicación del plan estratégico de comunicación se podrá potenciar el manejo de las herramientas informáticas de edición de los futuros profesionales de Comunicación Social, además se tienen las variables "Plan estratégico de comunicación y la insuficiencia en el manejo de las herramientas informáticas de edición" permitiendo la indagatoria y la formulación del diseño de un plan estratégico comunicacional para fortalecer los conocimientos sobre las insuficiencias y cómo solucionar los problemas que se evidencian.

El apoyo de todos los involucrados en este tema es fundamental, tanto estudiantes como docentes y las autoridades correspondientes, para el mejoramiento de las habilidades del profesional en lo referente a la edición de contenidos digitales. Actualmente, dentro del mundo de la comunicación, los estudiantes y profesionales que se desarrollan en ella, deben saber manejar la edición de su material de respaldo, es así que un reportero de medios televisivos debe poseer el conocimiento necesario para editar sus reportajes, el reportero de un medio radial deberá saber editar sus grabaciones de audio y un redactor de medios impresos tendrá que saber cómo editar sus notas para colocarlas dentro de los espacios respectivos ya sea su periódico, revista o libro.

Debido a esto, los medios de comunicación están en busca de profesionales en comunicación con plenos conocimientos sobre las herramientas tecnológicas que se utilizan en el ejercicio de su labor periodística. Así como lo exponía hace ya algún tiempo (Meyer, 2004, p.12) "Se necesitan buenos reporteros que puedan traer herramientas apropiadas para utilizarlas en situaciones constantemente cambiantes. En este ambiente, los periodistas que puedan hacer más de una cosa estarán en demanda".

Esa es la demanda de trabajos de calidad que los ecuatorianos exigen de los medios y de los profesionales de la comunicación. La ciudadanía en general ya está muy ligada a las TIC así lo demuestra el informe con los resultados de las estadísticas del (INEC, 2017) Instituto Nacional de Estadísticas y Censos del estudio que se realizó en noviembre de 2017, el cual se aplicó a 31.092 hogares a nivel nacional, regional, provincial, de nivel 
urbano y rural y que es una encuesta oficial que el Ecuador ejecuta consecutivamente sobre TIC entre esas cifras encontramos un incremento del 12, 1 puntos de los hogares posee un equipamiento de computadoras, tal como se observa en la figura 1. Para el año 2017,el acceso al nternet a nivel nacional se incrementó en 14,7 puntos más que en 2012; al igual que en área urbana, mientras que en la rural crece 11,8 puntos, estos datos se observan en la figura 2. Así también, 9 de cada 10 hogares en el país poseen al menos un teléfono celular, 9 puntos más que lo registrado en el 2012, datos que se visualizan en la figura 3.

Figura 1. Equipamiento tecnológico en el hogar.

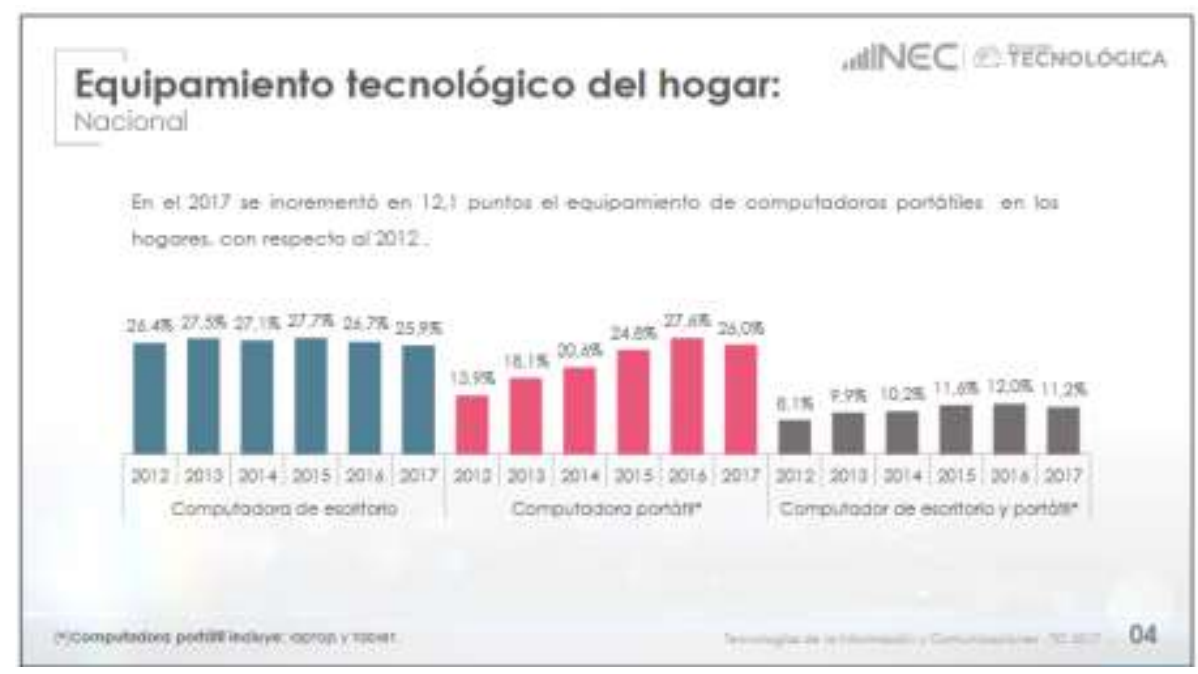

Fuente: (INEC, 2017).

Figura 2. Hogares con acceso a internet.

Hogares con acceso a Internet:

AINEC EFECNOLOCICA Nacional y area

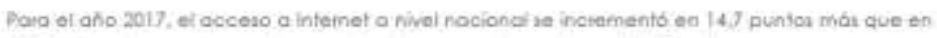
2012; al iqual que en drea ubona, mientran que en la murol erece 11,8 puntos.

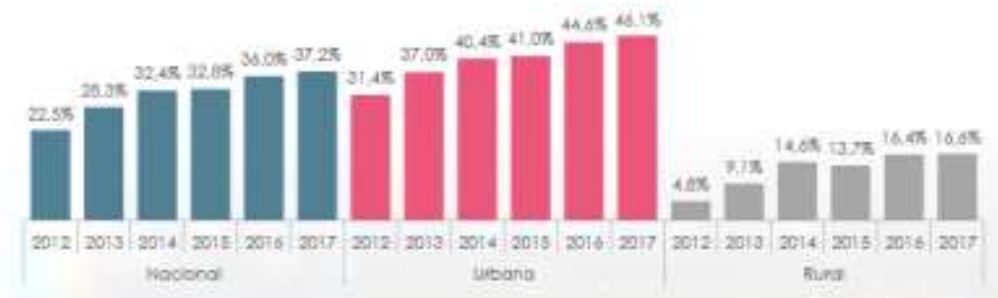

Fuente: (INEC, 2017). 
Figura 3. Hogares que tienen teléfono fijo y celular.

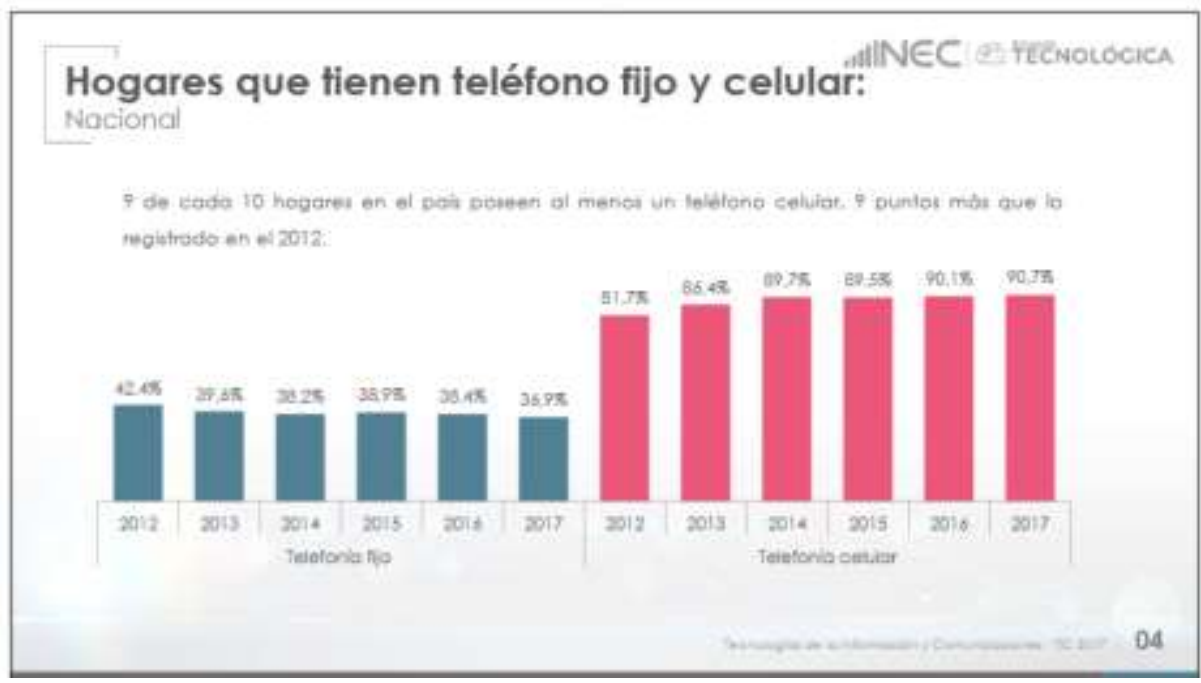

Fuente: (INEC, 2017).

Y es que la implementación de las nuevas tecnologías ha logrado crear nuevas formas de comunicación y del trabajo comunicacional en sí mismo, tal como lo menciona (Martín, 2002, p.35).

Lo que la trama comunicativa de la revolución tecnológica introduce en las sociedades no es tanto la cantidad inusitada de nuevas máquinas sino un nuevo modo de relación entre los procesos simbólicos, que constituyen lo cultural, y las formas de producción y distribución de los bienes y servicios.

El objetivo de esta investigación es caracterizar las deficiencias en la formación de los futuros profesionales de la Comunicación, relacionado con el uso de las TIC dentro del contexto educativo de los estudiantes de la carrera de Comunicación Social, siendo estas las herramientas informáticas de edición; sistematizar los contenidos sobre las herramientas de edición de audio, sonido y texto, tal como lo recomiendan (Flores Vivar \& Esteve Ramírez, 2009) en su libro Periodismo Web 2.0 "aceptar el nuevo paradigma de comunicación, adaptarse a los cambios que promueven las TIC y crear nuevos modelos informativos, como prioridades que los medios, profesionales y universidades deben asumir con rapidez". 
La comunicación siempre está en un entorno muy cambiante y por ello deben estar bien preparados para lograr realizar las actividades de una forma alineada a las nuevas tecnologías.

Así como lo señala la conclusión del V Congreso Internacional de Periodismo en Red que se llevó a cabo el 15 y 16 de noviembre de 2011 en España:

No se puede soslayar la realidad de que las tecnologías de la Información deben ser las compañeras inseparables de los periodistas que se desenvuelven en un entorno siempre cambiante" y recomendó "realizar profundos cambios en la formación de los profesionales de la comunicación quienes están inmersos en una era de transformaciones vertiginosas en todos los ámbitos de la información: desde las fuentes a las audiencias; desde los géneros tradicionales a las nuevas tecnologías.

Lo expresado anteriormente hace ver que en la actualidad las TIC, se han convertido en medios organizadores de la percepción del entorno ciudadano y fuente de nuevos objetivos y nuevas formas de entender. Como señala (Stephen, 1997):

Detrás de las placas de los discos, botones o palancas que conectan nuestra acción con una tecnología particular o con un sistema tecnológico, no solo hay un sistema mecánico o electrónico y un conjunto de circuitos, sino fundamentalmente un texto cultural que adquiere sentido de cuanto acontece para vincular la tecnología con la acción social y productiva.

La generación de estudiantes y profesionales de la denominada "Comunicación 2.0" deben asimilar de manera natural y nata el uso de herramientas tecnológicas, por lo que su implicación en la educación sugiere importantes beneficios en ellos, para esto el cambio y el aprendizaje continuo le permiten conocer las novedades que van surgiendo con el día a día, a nivel global.

\section{REVISIÓN TEÓRICA}

Las herramientas informáticas y en general todas las nuevas Tecnologías de la Información y de la Comunicación (TIC) han evolucionado aceleradamente en los últimos años, debido 
a su capacidad de interconexión a través del internet y de su implementación en casi todas las profesiones.

En la actualidad los estudiantes deben mantenerse a la par con la tecnología para avanzar en el desarrollo profesional y de la sociedad. Tal como lo indica (Jaramillo, 2003, p.25): “...la necesidad de ser creativo e innovador, profesional y flexible para poder enfrentar los cambios profundos y acelerados de la tecnología".

Esta nueva fase de desarrollo tiene gran impacto en lo que corresponde a la organización de la enseñanza y el proceso de aprendizaje en los estudiantes de todos los niveles. La incorporación de las herramientas informáticas en el entorno educativo y la adecuada utilización didáctica del mismo supone un reto sin precedentes con muchos beneficios, tanto para los docentes como en los futuros profesionales.

El sistema educativo no puede quedar al margen de los nuevos cambios. Debe atender a la formación de los nuevos profesionales y la incorporación de las nuevas tecnologías ha de hacerse con la perspectiva de favorecer los aprendizajes y facilitar los medios que sustenten el desarrollo de los conocimientos y de las competencias necesarias para la inserción en el ámbito social y lograr un profesional de calidad altamente competitivo.

Actualmente dentro del mundo de la comunicación, los estudiantes y profesionales que se desarrollan en ella, deben saber manejar la edición de su material de respaldo, es así que un reportero de medios televisivos debe poseer el conocimiento necesario para editar sus reportajes, el reportero de un medio radial deberá saber editar sus grabaciones de audio y un redactor de medios impresos tendrá que saber cómo editar sus notas para colocarlas dentro de los espacios respectivos de su periódico, revista o libro.

Debido a esto, los medios de comunicación están en busca de profesionales con plenos conocimientos sobre las herramientas tecnológicas que se utilizan en el ejercicio de su labor periodística. Así como lo exponía hace ya algún tiempo (Meyer, 2004): "Se necesitan buenos reporteros que puedan traer herramientas apropiadas para utilizarlas en situaciones constantemente cambiantes. En este ambiente, los periodistas que puedan hacer más de una cosa estarán en demanda". 


\section{Las herramientas tecnológicas en la formación profesional}

Según el sitio web (EcuRed ${ }^{1}$ ) las herramientas informáticas de edición son programas computacionales creados para facilitar trabajos específicos en los diferentes campos profesionales, dichos programas pertenecen al software de la computadora.

La denominación del software de un computador según (EcuRed.cu) equivale al espíritu de una computadora, el uso y el aprovechamiento de este y todos aquellos beneficios que otorga el entorno del computador, depende del software, ayudando a los usuarios la creación de programas informáticos que apoyen con las labores cotidianas, así como las personales, como las generales, las industriales y organizacionales, el software en su variedad de clasificación es el componente fundamental como enlace entre el usuario y la computadora, su evolución desde un inicio se denotaba con poca interés pero con el transcurrir de los años ha tomado un papel muy importante entre los programadores y creativos de los software tanto de aplicación como operativos, absolutamente todo aquello que se observa digitalizado en una computadora corresponde al software de la misma, denominándolo de alguna manera, como "Herramientas informáticas".

\section{Software de sistema}

Tal como lo define el sitio web (glosario de informática e internet ${ }^{2}$ ) es el sistema básico o software operativo. Este es la unión de programas cuya finalidad es ayudar en el manejo de la computadora y logra que se utilice más eficientemente. Este también organiza y designa a las diferentes herramientas del sistema, también conocido como hardware.

\section{Software de Aplicación}

Las nuevas herramientas informáticas de edición proporciona a los estudiantes de comunicación y diseño un nuevo reto y es de estar a la par en la creación de la siguiente generación de contenidos digitales.

\footnotetext{
${ }^{1}$ https://www.ecured.cu/EcuRed:Enciclopedia_cubana

2 https://www.internetglosario.com/
} 
Tal como lo orienta (Ramonet, 2014):

Los adelantos tecnológicos futuros de las herramientas informáticas dentro del periodismo son muy prometedores, miles de diseñadores, desarrolladores y líderes de negocios se encuentran en la tarea de descubrir un nuevo mundo de opciones positivas para lograr la expansión de los contenidos y de las aplicaciones que utilicen múltiples pantallas, que incorporen los contenidos digitales y comerciales y los programas profesionales para diseño y desarrollo, que ya no estarán dirigidas a captar el entorno comunicacional de los medios de masas sino a la nueva tendencia de las "masas de medios".

Así como también lo expresa (Lynch, 2014): "Todos los días, los adelantos tecnológicos transforman la manera de trabajar, aprender, comunicarse, en fin: la manera en que nos expresamos", Kevin Lynch, director de tecnología de Adobe. Estas herramientas informáticas ayudarán a las organizaciones a tener éxito en un nuevo mundo "multipantalla" donde se profundizará en el diseño en múltiples tipos de medios y pantallas.

El software de aplicación es definido por el sitio web (glosario de informática e internet) como aquellos programas que controlan y optimizan el manejo de la computadora, define la interfaz primaria e importante entre el operador y la computadora, estos logran que el operador pueda manejar de modo fácil y simple las complicadas herramientas del sistema hardware, cumpliendo acciones que para el operador serían difíciles e incluso no probables de realizar, y estas hacen como mediador entre el operador y el software de aplicación.

En el hardware es donde se tienen las herramientas informáticas diseñadas para que realicen las acciones en los diferentes entornos o áreas profesionales. Ejemplo: los programas de edición de información en la Comunicación Social, los programas de diseño en Arquitectura, los programas virtuales en Medicina, los programas de contabilidad para Administración, etc.

En la actualidad tanto el estudiante como el profesional de la Comunicación Social y periodismo deben poseer el conocimiento y el manejo de tres programas fundamentales que utilizan los medios de comunicación para poder ejercer su labor diaria en cualquiera de los campos comunicacionales: 
Adobe Audition (Radio), según lo expresado en la página web Ecured.cu³:

Es un programa para edición y grabación de música digital, con el cual puedes obtener una gran variedad de opciones, y puedas obtener y realizar un trabajo de calidad y obtener un buen producto. Diseñado para los profesionales de audio y video más exigentes, ofrece funciones avanzadas de mezcla, edición y efectos de sonido.

Adobe Premiere (Televisión), según la conceptualización dada en la página web Ecured.cu: "es una aplicación en forma de estudio destinada a la edición de video y desarrollo web". Adobe InDesing (Medios Impresos: (Periódicos y Revistas), según la conceptualización dada en la página web Ecured.cu: "es una aplicación para la composición digital de páginas desarrollada por la compañía Adobe Systems y dirigida a maquetadores profesionales".

Revisada la actual malla curricular de la carrera de comunicación social aparece una materia de nombre "Computación", en segundo semestre, y luego de realizar una evaluación diagnóstica a los estudiantes si en esta asignatura veían aquellos contenidos necesarios para el desarrollo de sus destrezas en campos tales como edición de fotografía, edición de audio, edición de video, postproducción, ellos estiman que es muy corto período lo cual no es el suficiente para desarrollar las destrezas, que son consideradas como muy importantes en el momento de enfrentarse al mundo laboral.

Se debe tomar como referencia que en Ecuador según datos proporcionados por el INEC, en su cuarta encuesta oficial ejecutada consecutivamente sobre las Tics. Indica que el $22.8 \%$ de los hogares posee un ordenador personal, el 53.8\% de la población a nivel nacional usa internet y el $78.8 \%$ de las personas posee teléfonos móviles, así como que un $40 \%$ de quienes utilizan internet lo hacen para actividades relacionadas con la educación y el aprendizaje. Este estudio, que se realizó en diciembre de 2011, se aplicó a 21.768 hogares a nivel regional, provincial y nacional tanto en el nivel urbano como en el rural.

Por tal referencia, la Facultad de Comunicación Social debe brindar todas las facilidades, tanto pedagógicas como de infraestructura, para el proceso de enseñanza - aprendizaje de las nuevas tecnologías dentro de sus campos de estudios, siempre orientados hacia las necesidades de sus estudiantes frente a los retos del campo laboral.

\footnotetext{
${ }^{3}$ https://ecured.cu/Adobe_Audition
} 
En la actualidad los estudiantes, de todas las carreras deben poseer las habilidades necesarias para ser competitivo dentro del mundo laboral, esto sugiere tener una preparación de vanguardia y alineada a las necesidades que demanda la sociedad. En la actualidad el internet y las nuevas tecnologías abarcan la mayoría de las profesiones, los estudiantes universitarios deben poner su máxima atención hacia el manejo de todo lo que corresponde al uso de las TIC, los docentes deben preparar a sus alumnos para poder desenvolverse con total normalidad cuando entren a ejercer su profesión.

En tal sentido, se han hecho grandes esfuerzos por partes de las unidades educativas por estar a la par tecnológicamente con lo que demanda el entorno pero no se ha logrado obtener buenos resultados, notándose cómo los estudiantes tienen problemas cuando al salir de la universidad van a ejercer las labores diarias dentro del campo de su profesión y se ven limitados en el manejo de dichas tecnologías y puntualmente en el uso de las herramientas informáticas.

\section{MATERIALES Y MÉTODOS}

Se ha utilizado un diseño cuantitativo donde la descripción es el método fundamental, se implementaron además los métodos teóricos para interpretar los datos empíricos encontrados; profundizar en las relaciones esenciales y cualidades fundamentales de los procesos no observables directamente; elaborar conceptos y brindar conclusiones.

Histórico y Lógico: posibilitó el estudio y evolución de las herramientas tecnológicas hasta convertirse en idóneas para la labor periodística actual.

Análisis y Síntesis: permitió el análisis de diferentes materiales bibliográficos acerca de los principales elementos de las herramientas informáticas de edición además evaluar las dificultades y descomponer el objeto-fenómeno (manejo de herramientas de edición).

Inducción y Deducción: facilitó realizar un análisis del problema mediante la contrastación empírica, determinando inferencias que sirvieron a su vez para identificar las causas que lo originaron. Llegar a generalizaciones a partir del estudio de la muestra y a la vez pasar de un conocimiento general a uno específico. 


\section{Población y muestra}

La población está compuesta por los 161 estudiantes del sexto semestre de la Carrera de Comunicación Social, sección Nocturna de FACSO. La muestra la comprenden los 80 estudiantes que constituye el $50 \%$ por ciento de la población por lo que se puede afirmar que es representativa, se clasifica como Intencional no probabilística porque fue seleccionada por los autores de acuerdo con sus intereses.

En la investigación previa se pudo conocer que la muestra recibió de acuerdo a la malla vigente para ese período lectivo las materias de Computación, Producción Audiovisual y Comunicación Multimedia I, así como los talleres de Periodismo digital y Producción periodística por lo tanto pueden señalar la forma en que se está llevando a cabo su proceso de aprendizaje y sus expectativas sobre las materias recibidas.

\section{Técnicas utilizadas en la investigación}

Las técnicas que los autores utilizó en la investigación para obtener la información requerida fueron:

La Observación: Esta técnica permitió visualizar al objeto de investigación en su proceso educacional, con visitas a clases, en los estudios de televisión, de prensa y de radio

La Encuesta: que se aplicó a una muestra intencionada y variada, dirigida a recopilar datos valorativos, la misma que contó con el apoyo de un cuestionario, el cual sirvió de enlace entre los objetos de la investigación y la realidad del estudiantado.

Métodos estadísticos: Técnica utilizada mediante el empleo de por cientos (Análisis porcentual) y de gráficos que ilustraron de manera más clara los resultados obtenidos en los instrumentos aplicados.

\section{Instrumentos}

El cuestionario: dirigido a los estudiantes del sexto semestre de la carrera de Comunicación Social, elaborado con preguntas objetivas y que permitió recabar información sobre las variables de estudio: 


\section{ANÁLISIS DE LOS RESULTADOS}

En la indagatoria se realizaron encuestas a los estudiantes, presentamos a continuación tres ejemplos que lo representa:

¿Qué nivel de conocimiento sobre las herramientas informáticas de edición posee?

Tabla 1. Nivel de conocimiento de las herramientas informáticas de edición.

\begin{tabular}{ccc}
\hline OPCIONES & SEÑALADAS & PORCENTAJE \\
\hline Alto & 17 & $21 \%$ \\
Medio & 16 & $20 \%$ \\
Bajo & 31 & $39 \%$ \\
Nulo & 16 & $20 \%$ \\
\hline Total: & $\mathbf{5 4}$ & $\mathbf{1 0 0} \%$ \\
\hline
\end{tabular}

Fuente: Encuesta a los estudiantes de la carrera Comunicación Social FACSO.

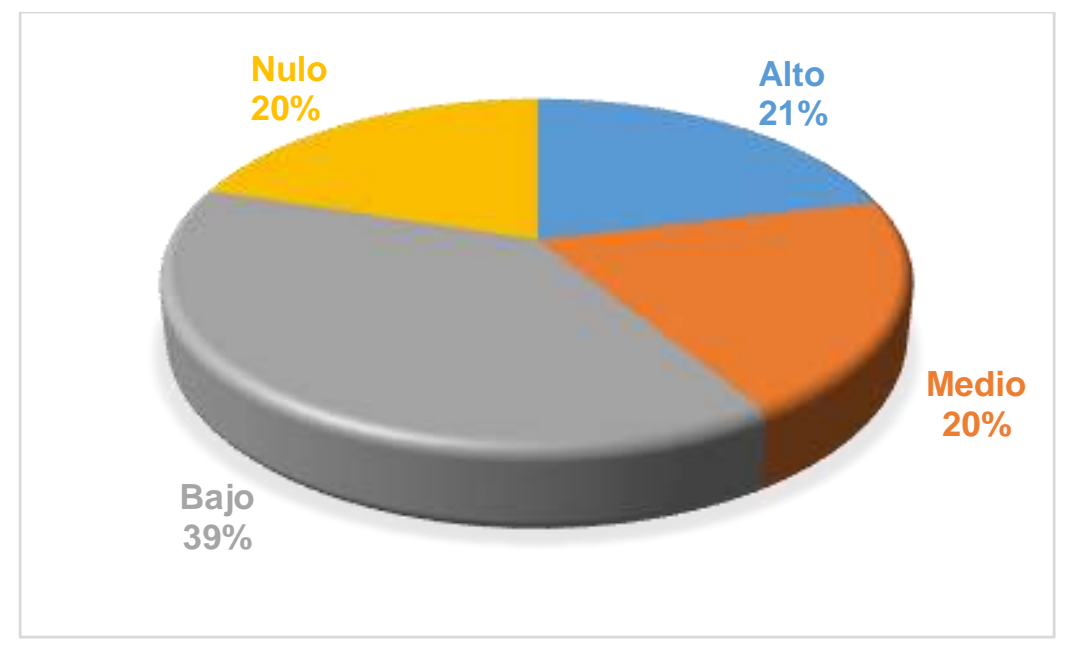

Figura 4. Nivel de conocimiento de las herramientas informáticas de edición. Fuente: Elaboración propia.

Análisis e interpretación: De los 80 estudiantes encuestados que constituyen el 100\% de la muestra, 31 estudiantes equivalentes al 35\% indican que tienen un nivel bajo de conocimiento sobre las herramientas informáticas de edición, 17 estudiantes equivalentes al $21 \%$ indican que tienen un nivel alto, 16 estudiantes equivalentes al $20 \%$ indican que 
tienen un nivel medio y bajo de conocimiento sobre las herramientas informáticas de edición.

Con los resultados mostrados por la encuesta se puede interpretar que la muestra a pesar de haber realizado trabajos de edición en su mayoría posee un conocimiento con nivel bajo sobre las herramientas informáticas, denotando que se necesita profundizar en la enseñanza sobre el uso de estas herramientas.

¿Qué programa de edición se utiliza para el diseño de documentos que eventualmente vas a imprimir de manera estándar o quieres visualizar en línea?

Tabla 2. Programa de edición para el diseño de documentos.

\begin{tabular}{ccc}
\hline OPCIONES & SEÑALADAS & PORCENTAJE \\
\hline WordPress & 16 & $20 \%$ \\
InDesing & 19 & $24 \%$ \\
Instapaper & 11 & $14 \%$ \\
Blanco & 34 & $42 \%$ \\
\hline Total: & $\mathbf{8 0}$ & $\mathbf{1 0 0} \%$ \\
\hline
\end{tabular}

Fuente: Encuesta a los estudiantes de la carrera Comunicación Social FACSO.

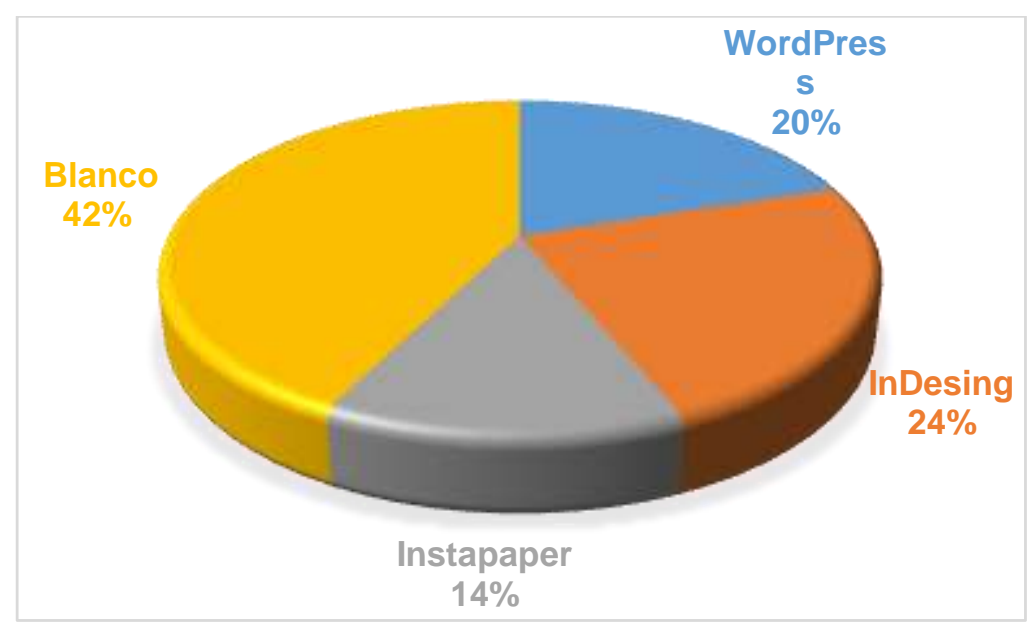

Figura 5. Programa de edición para el diseño de documentos. Fuente: Elaboración propia.

Análisis e interpretación: De los 80 estudiantes encuestados que constituyen el $100 \%$ de la muestra, 34 estudiantes equivalentes al 42\% dejaron la pregunta en blanco, 19 estudiantes equivalentes al $24 \%$ indican que es InDesing, 16 estudiantes equivalentes al $20 \%$ indican que es WordPress y 11 estudiantes equivalentes al 14\% indican que el programa de edición 
que les permite diseñar documentos para su posterior impresión y visualización es Instapaper.

Los resultados de la encuesta revelaron que solo un cuarto de la muestra poseen el conocimiento sobre qué programa de edición permite diseñar documentos para su posterior impresión y visualización, fundamentando la necesidad de socializar los contenidos del programa InDesing.

¿Qué programa de edición me permite trabajar en forma de estudio de sonido y en un entorno de edición mezclado multipista?

Tabla 3. Programa de edición de sonido.

\begin{tabular}{|c|c|c|}
\hline OPCIONES & SEÑALADAS & PORCENTAJE \\
\hline Audio Hijackpro & 10 & $12 \%$ \\
\hline Wisestamp & 6 & $7 \%$ \\
\hline Audition & 38 & $48 \%$ \\
\hline Blanco & 26 & $33 \%$ \\
\hline Total: & 80 & $100 \%$ \\
\hline
\end{tabular}

Fuente: encuesta a los estudiantes de la carrera Comunicación Social FACSO.

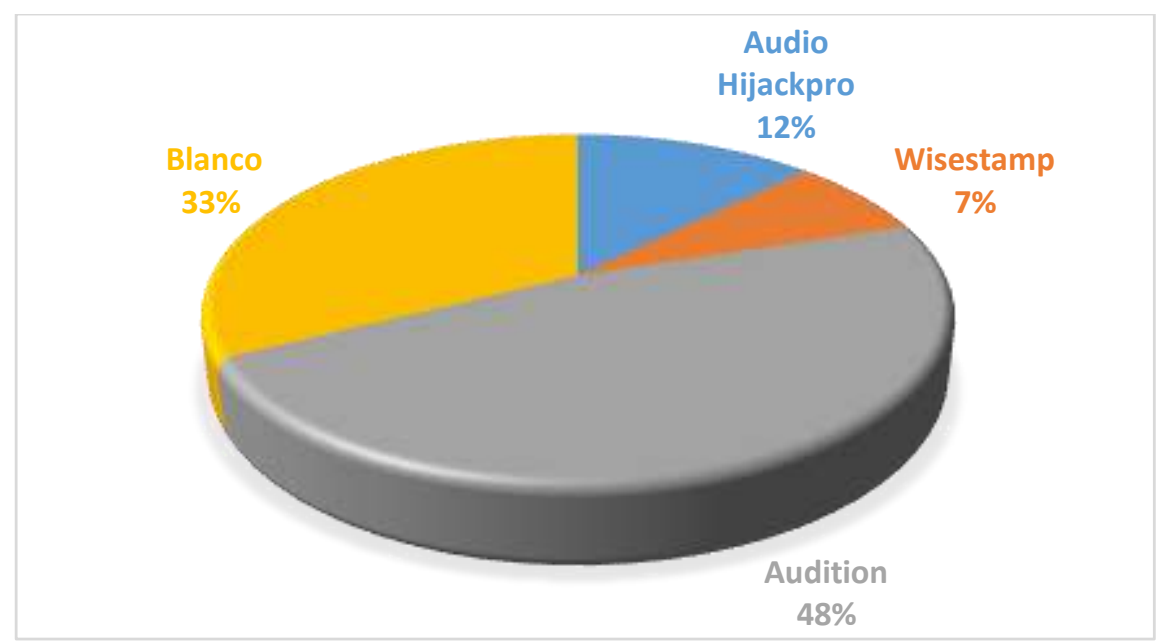

Figura 6. Programa de edición de sonido.

Fuente: Elaboración propia.

Análisis e interpretación: De los 80 estudiantes encuestados que constituyen el 100\% de la muestra, 5 estudiantes equivalentes al $48 \%$ indican que el programa de edición que me permite trabajar en forma de estudio de sonido y en un entorno de edición mezclado 
multipista es el Audition, 26 estudiantes equivalentes al 33\% dejaron la pregunta en blanco, 10 estudiantes equivalentes al 12\% indican que es Audio Hijackpro y 6 estudiantes equivalentes al $7 \%$ indican que es Wisestamp.

Los autores con base en los resultados mostrados por la encuesta interpreta que la mitad de la muestra posee el conocimiento sobre el programa de edición de audio Adobe Audition demostrando la necesidad de reforzar dicho conocimiento en la otra mitad de la muestra.

\section{Interpretación final}

La muestra aunque afirma que realiza trabajos de edición con herramientas informáticas califica su conocimiento sobre las mismas como bajo, denotando falencias en lo que respecta a la edición de texto e imágenes, puntualizando el hecho de no recibir ese conocimiento en las clases, limitando su desarrollo y creatividad en productos periodísticos digitales y en sus competencias para el ejercicio profesional en el entorno comunicacional. Justificando en base a los resultados obtenidos la necesidad de fortalecer los conocimientos sobre las herramientas informáticas de edición en los estudiantes.

Como parte del estudio realizado a la malla curricular de la carrera se pudo constatar que la asignatura computación no logra establecer los parámetros necesarios para el desarrollo de las habilidades en las herramientas de edición para el profesional que se pretende formar, todo ello permite formular las siguientes conclusiones.

\section{PROPUESTA}

\section{Diseño de plan estratégico de comunicación}

Después de realizar la investigación referente a la insuficiencia en el manejo de herramientas informáticas de edición el autor plantea la elaboración de un Plan estratégico de comunicación que favorezca el aprendizaje y dominio de dichas herramientas en los estudiantes de la carrera de comunicación social de la Universidad Estatal de Guayaquil. La carrera de comunicación social comprende ocho semestres de preparación universitaria; donde se educan a estudiantes comprometidos con la facultad, la universidad y la sociedad, los cuales logran obtener los conocimientos y capacidades que fortalecen sus habilidades para satisfacer las diferentes necesidades y requerimientos de las labores profesionales; 
pero se debe profundizar en las actividades prácticas de dichos conocimientos para que se desarrolle en los estudiantes las competencias específicas en el manejo de las herramientas informáticas de edición y de esta manera crear en ellos esa motivación para innovar y crear sus propios contenidos periodísticos, colocando en ellos ese sello personal que los caracterizará en el ejercicio de su profesión.

En la carrera existen asignaturas y talleres donde se brinda conocimiento sobre el periodismo digital y las herramientas informáticas, en contraste, este no brinda ese estudio práctico para vincular las actividades diarias de los estudiantes con el uso de las herramientas informáticas de edición, el cual permita generar ese interés por completo, sobre las capacidades que pueden desarrollar y aplicar para realizar trabajos profesionales de calidad.

En la actualidad los estudiantes deben mantenerse a la par con la tecnología para avanzar en el desarrollo de su profesión y de la sociedad, dada la necesidad de ser creativo e innovador, y flexible para poder enfrentar los cambios profundos y acelerados de la tecnología.

Hoy en día se ve cómo los jóvenes interactúan con imágenes, audio y texto desde sus teléfonos inteligentes, laptops, tablets y demás aparatos electrónicos y todo esto lo perciben como un hobby para su entretenimiento personal, no lo ven como una forma de aprendizaje para ampliar sus conocimientos a la hora de hacer sus tareas estudiantiles, no lo ven como una herramienta funcional que perfeccionaría el ámbito profesional y académico dentro de sus vidas.

Bajo estos argumentos existe la importancia de potenciar el conocimiento de las herramientas informáticas de edición en los estudiantes brindándoles la libertad en el momento de diseñar con su propia creatividad productos comunicacionales digitales, demostrando sus capacidades para un desarrollo tecnológico.

Socializar la información relevante sobre el uso de las herramientas informáticas de edición con la implementación de un Plan estratégico de Comunicación, para el reforzamiento de las habilidades de los estudiantes del sexto semestre de la carrera de Comunicación Social de la facultad de Comunicación Social de la Universidad Estatal de Guayaquil. 


\section{CONTENIDO DE LA PROPUESTA}

El Plan estratégico de comunicación consiste en la socialización mediante material de apoyo didáctico y la realización de talleres prácticos que se brindarán en un espacio físico que cuente con la infraestructura adecuada en el que los estudiantes puedan recibir la información relevante y prácticas sobre las herramientas informáticas de edición, llevar el conocimiento básico sobre periodismo digital y sus herramientas a las aulas y de esta forma fomentar el aprendizaje actual y efectivo para conseguir una transformación importante en sus trabajos digitales.

Ubicación: Universidad Estatal de Guayaquil, Facultad de Comunicación Social, Guayas, Ecuador.

Beneficiarios: Estudiantes del sexto semestre de la carrera de Comunicación Social.

Factibilidad: La propuesta es factible porque la carrera de Comunicación Social, cuenta con una infraestructura adecuada para el aprendizaje de estas tecnologías; la Facultad de Comunicación Social tiene un laboratorio de computación donde permanentemente se facilita la conexión de internet con una señal abierta para crear productos comunicacionales digitales.

Tiempo estimulado para la ejecución: Dos meses.

Equipo técnico responsable: Docentes del área informática y Análista TIC de la FACSO.

\section{Plan de acción}

Tabla 4. Plan de acción.

\begin{tabular}{|c|c|c|c|c|}
\hline FASES & METAS & ACTIVIDADES & RESPONSABLES & TIEMPO \\
\hline $\begin{array}{l}\text { APROBACIÓN } \\
\text { DEL PROYECTO. }\end{array}$ & $\begin{array}{l}\text { Puesta en } \\
\text { marcha } \\
\text { de la Propuesta. }\end{array}$ & $\begin{array}{l}\text { Exposición del } \\
\text { Proyecto. }\end{array}$ & $\begin{array}{l}\text { Docentes del área } \\
\text { informática y Analista } \\
\text { TIC de la FACSO. }\end{array}$ & Una semana \\
\hline $\begin{array}{l}\text { GESTIÓN DEL } \\
\text { ESPACIO } \\
\text { FÍSICO } \\
\text { PARA LOS }\end{array}$ & $\begin{array}{l}\text { Contar con un } \\
\text { aula con la } \\
\text { infraestructura. }\end{array}$ & $\begin{array}{l}\text { Adecuación de } \\
\text { ambientes: } \\
\text { Infraestructura e } \\
\text { Equipamiento. }\end{array}$ & $\begin{array}{l}\text { Docentes del área } \\
\text { informática y Analista } \\
\text { TIC de la FACSO. }\end{array}$ & Una semana \\
\hline
\end{tabular}




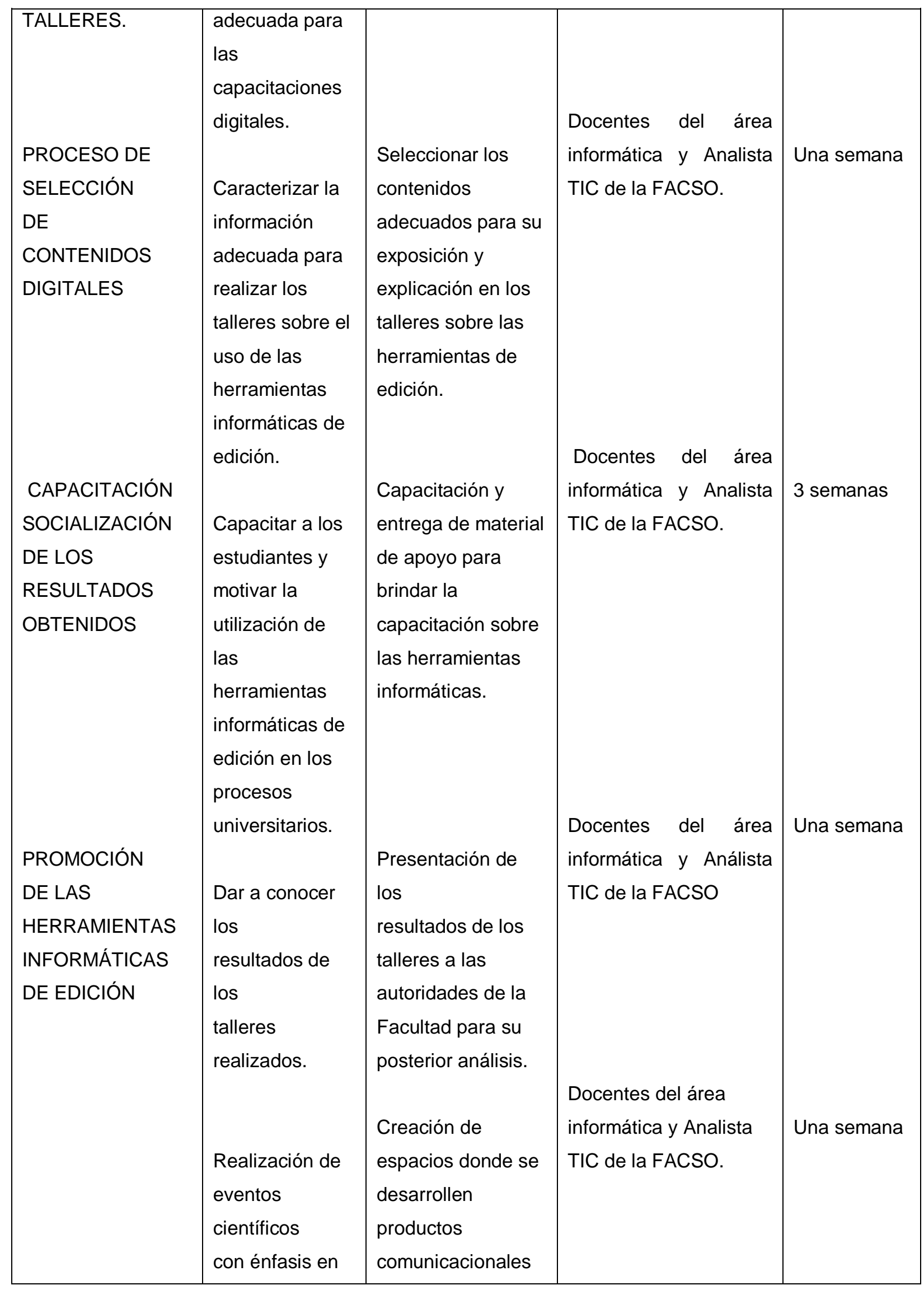




\begin{tabular}{|l|l|l|l|l|}
\hline & $\begin{array}{l}\text { trabajos con las } \\
\text { Herramientas } \\
\text { informáticas de } \\
\text { edición. }\end{array}$ & $\begin{array}{l}\text { basados en los } \\
\text { contenidos de las } \\
\text { capacitaciones. }\end{array}$ & & \\
\end{tabular}

Fuente: Elaboración propia.

\section{Calendario de talleres}

Tabla 5. Cronograma de ejecución de talleres.

\begin{tabular}{|c|c|c|c|}
\hline \multicolumn{4}{|c|}{ ENERO } \\
\hline SEMANA 1 & $\begin{array}{l}\text { Taller de Adobe } \\
\text { Audition. }\end{array}$ & $\begin{array}{l}\text { En este taller se enseñará a editar todo tipo de } \\
\text { audios, se aplicarán efectos de sonido, se } \\
\text { darán las pautas para mezclar audios, se } \\
\text { enseñará a agregar filtros de ruido en las } \\
\text { pistas musicales, a utilizar el sistema } \\
\text { multipistas, compilar y editar una banda } \\
\text { sonora, limpiar el audio de una película, grabar } \\
\text { y mezclar proyectos sonoros. }\end{array}$ & $\begin{array}{l}\text { C1 } \\
\text { C2 } \\
\text { C3 }\end{array}$ \\
\hline SEMANA 2 & $\begin{array}{l}\text { Taller de Adobe } \\
\text { Premiere. }\end{array}$ & $\begin{array}{l}\text { En este taller se brindarán los conceptos } \\
\text { teóricos y prácticos para crear vídeos en } \\
\text { distintos formatos como AVI y MP4, también se } \\
\text { crearan transiciones entre vídeos, para } \\
\text { dejarlos en uno sólo, lograr hacer } \\
\text { transparencias sobre imágenes y vídeo, editar } \\
\text { y técnicas relacionadas a los vídeos e } \\
\text { incorporar los diferentes efectos especiales a } \\
\text { los productos audiovisuales. }\end{array}$ & $\begin{array}{l}\text { C1 } \\
\text { C2 } \\
\text { C3 }\end{array}$ \\
\hline SEMANA 3 & $\begin{array}{l}\text { Taller de Adobe } \\
\text { InDesing. }\end{array}$ & $\begin{array}{l}\text { Aquí se logrará enseñar la administración del } \\
\text { color así como a gestionar la reutilización hacia } \\
\text { los formatos HTML y XML, operar los apartados } \\
\text { de control tipográfico, el manejo de imágenes, } \\
\text { las herramientas de diseño y productividad, así } \\
\text { como la generación de tablas y la exportación } \\
\text { de archivos a PDF. }\end{array}$ & $\begin{array}{l}\text { C1 } \\
\text { C2 } \\
\text { C3 }\end{array}$ \\
\hline
\end{tabular}

Fuente: Elaboración propia. 


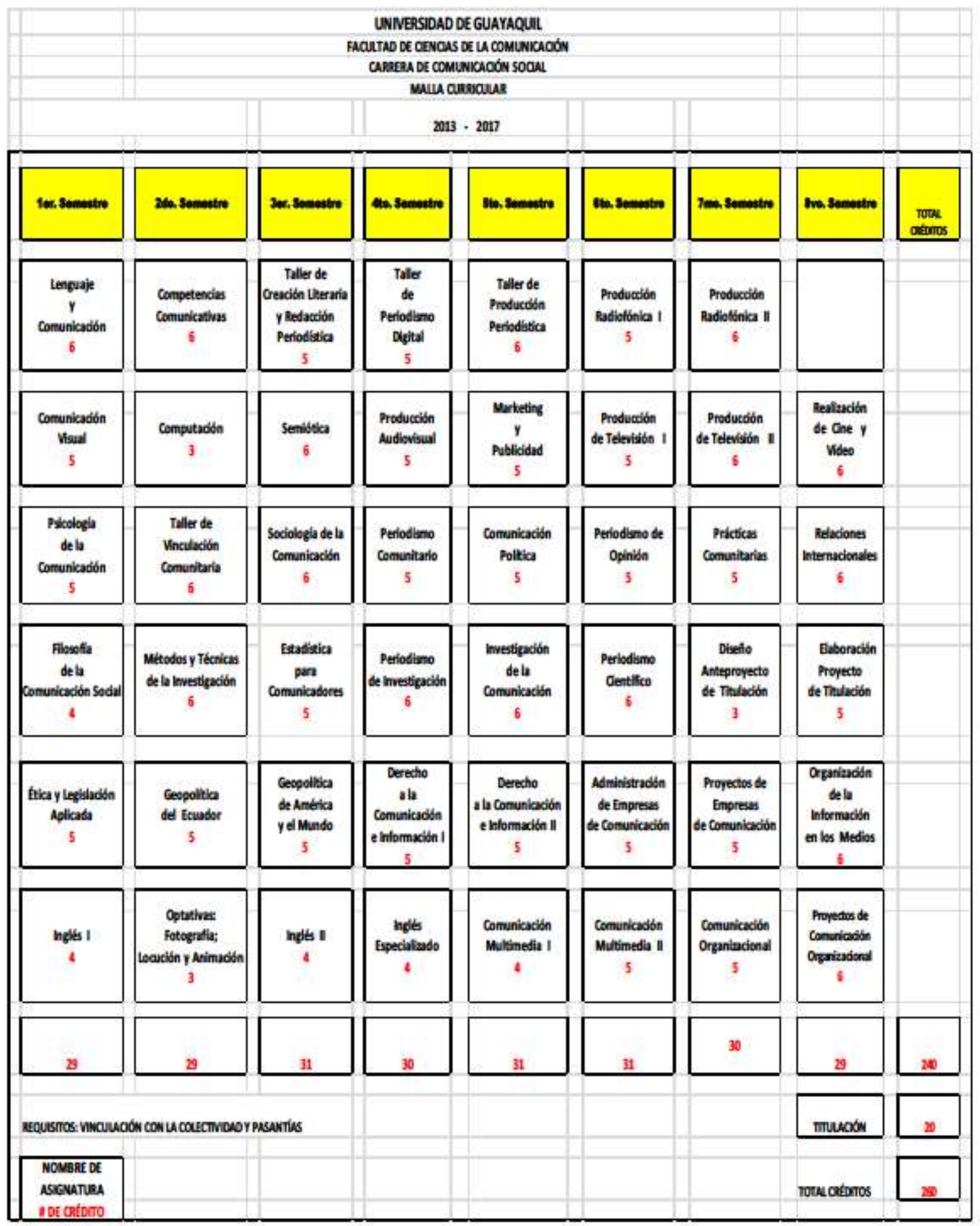

Figura 6. Malla curricular de la carrera Comunicación Social de la Universidad de Guayaquil.

Fuente: Secretaría de la carrera Comunicación Social de la Universidad de Guayaquil. 


\section{CONCLUSIONES}

El nivel de conocimiento teórico práctico es un factor determinante en el desarrollo de las prácticas pre profesionales y en el dominio de las herramientas informáticas de edición, audio, sonido y texto.

Se recomienda profundizar con prácticas el uso de las herramientas informáticas de edición en las materias fundamentales de la carrera, donde se les permita a los estudiantes de comunicación social poner a prueba los conocimientos que van adquiriendo en las clases, estas prácticas se deben realizar desde los primeros semestres de la carrera, para potenciar las capacidades de los estudiantes corrigiendo las posibles falencias durante el tiempo universitario y obtener las competencias necesarias para el óptimo desempeño laboral dentro del mundo comunicacional.

Diseñar espacios dentro de los canales oficiales tanto convencionales como digitales de la facultad, para difundir información relevante sobre el manejo y aplicación de las herramientas informáticas de edición con el fin de brindar apoyo a los estudiantes de Comunicación Social, garantizando su participación en todos los procesos de trabajo, donde se pueda editar contenidos para su posterior publicación en esos mismos espacios. Realizar un estudio de la malla curricular para ajustar las necesidades evidenciadas en esta investigación. De mantenerse la estructura de la malla vigente, se revisen los contenidos de los syllabus de la asignatura de computación.

Uno de los criterios de evaluación de revisión del presente trabajo es: "Se transfieren los resultados a los grupos de interés." Para poder cumplir con este criterio y con este objetivo específico, se recomienda socializar los resultados de la encuesta con un grupo de trabajo compuesto de los estudiantes de sexto semestre, de profesionales del área informática y de responsables administrativo de la FACSO con el afán de incluir la colaboración del cuerpo estudiantil en la propuesta del diseño del plan cominicacional y presentar el plan como solución al problema. 


\section{REFERENCIAS BIBLIOGRÁFICAS}

Canga, J. (2001). Periodismo e internet: Nuevo medio, vieja profesión. Revista Estudio sobre el mensaje periodístico. Núm. 7, 2001. Recuperado de: https://revistas.ucm.es/index.php/ESMP/article/view/ESMP0101110033A

Fainholc, B. (1997). Nuevas Tecnologías de la Información y la Comunicación en la Enseñanza. Argentina: Aique Grupo Editor S.A

Flores, J., \& Esteve, F. (2009). Periodismo Web 2.0. Madrid: Fragua.

INEC (2017). Encuesta sobre TIC. Recuperado de http://www.ecuadorencifras.gob.ec/documentos/webinec/Estadisticas Sociales/TIC/2017/Tics\%202017 270718.pdf

Jaramillo, E. (2003). Desafios de la Radio en el nuevo milenio. Quito: Quipus.

Mardones, J.M. (1991). Filosofía de las ciencias humanas y sociales. Materiales para una fundamentación científica. Barcelona: Anthropos.

Martín, J. (2002). La educación desde la comunicacion. México: Gustavo Gili.

Meyer, F. (2004). El periódico desaparecido: Salvando el periodismo en la era de la información. Barcelona: Bosch.

Ramonet, I. M. (2014). La explosión del periodismo. Soria, España: Clave intelectual.

Stephen, H. (1997). La fuerza cultural de los sistemas tecnológicos, en innovación tecnológica y procesos culturales. México: Ediciones cientificas universitariasUNAM-F.C.E. 